\title{
Toroidal modelling of RMP response in ASDEX-Upgrade: coupling between field pitch aligned response and kink amplification
}

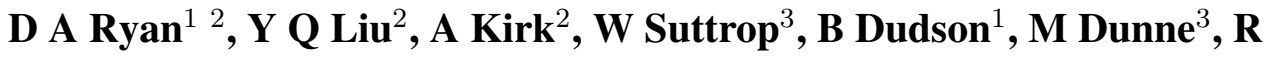 \\ Fischer $^{3}$, J C Fuchs ${ }^{3}$, M Garcia-Munoz ${ }^{3}{ }^{4}$, B Kurzan ${ }^{3}$, P Piovesan $^{5}$, M \\ Reinke $^{1}$, M Willensdorfer ${ }^{3}$ and the ASDEX-Upgrade team ${ }^{3}$ \\ ${ }^{1}$ York Plasma Institute, Department of Physics, University of York, Heslington, York, YO10 \\ 5DQ, UK \\ ${ }^{2}$ CCFE, Culham Science Centre, Abingdon, Oxfordshire, OX14 3DB, UK \\ ${ }^{3}$ Max Planck Institute for Plasma Physics, Garching, Germany \\ ${ }^{4}$ FAMN Department, Faculty of Physics, University of Seville, 41012 Seville, Spain \\ ${ }^{5}$ Consorzio RFX, Euratom-ENEA Association, Padova, Italy
}

\begin{abstract}
Using the MARS-F code (Liu et al 2000 Phys. Plasmas 7 3681), the single fluid resistive MHD plasma response to applied $n=2$ resonant magnetic perturbations is computed, for a plasma discharge in the ASDEX-Upgrade tokamak. The computation predicts strong kink amplification, as previously predicted in DIII-D (Haskey et al 2014 Plasma Phys. Control. Fusion 56 035005), which is strongly dependent on the toroidal phase shift between the upper and lower coils, $\Delta \phi_{u l}$. In particular, edge localised low $n$ peeling modes with poloidal mode numbers just above pitch resonance - a subset of the kink response - are amplified. The robustness of the amplified peeling response with respect to truncation of the $\mathrm{X}$ point is investigated, by recomputing the plasma response for a range of edge geometries. It is found that the computed peeling response, when plotted against the safety factor, is not sensitive to the numerical truncation near the $\mathrm{X}$ point. It is also predicted that near the plasma edge where resistivity is large, the pitch resonant components are finite and also strongly dependent on $\Delta \phi_{u l}$. A previous proposal that the amplified peeling response may indirectly drive the pitch aligned components by spectral proximity (Lanctot et al 2013 Nuclear Fusion 53 083019), is investigated by applying magnetic perturbations of a single poloidal harmonic, as a boundary condition at the plasma edge. It is found that poloidal harmonic coupling causes harmonics to couple to and drive harmonics directly beneath them spectrally, and also that the pitch aligned components can be driven by this mechanism. As a refinement, the amplified peeling response is simulated in relative isolation by using a filtered boundary condition. In this model, the peeling response drives the pitch aligned components to be comparable to their values in response to the RMP coils. This suggests that it is quite possible that the amplified low $n$ peeling response can drive the pitch aligned components in some circumstances, which would alter the coil configuration for optimum plasma stochastization, with implications for ELM control by RMPs.
\end{abstract}




\section{Introduction}

Edge Localised Modes (ELMs) are bursty plasma instabilities, which occur repetitively in High Confinement mode tokamak plasmas [1]. They are common in modern tokamaks, but usually do not pose a problem at these machine scales. However, extrapolations to ITER suggest that if ELMs were allowed to occur unmitigated, then machine components would be at risk of damage [?].

Since it was discovered that ELMs can be strongly mitigated or completely suppressed by the application of Resonant Magnetic Perturbation fields [2], intensive research has been underway attempting to expand the parameter spaces of ELM suppression and mitigation, and to understand the underlying mechanisms. To date, ELM suppression or mitigation by RMPs has been achieved on DIII-D [2], KSTAR [3], JET [4], MAST [5] and ASDEX-Upgrade [6]. However, the interaction between tokamak plasmas, ELMs and RMPs is highly complex, and a robust predictive theory of ELM suppression by RMPs is currently lacking [7]. Such a theory is urgently required, in order to predict the efficacy of the ITER ELM coils.

Stochastic Transport Theory [8] is one of several working theories of ELM suppression by RMPs, though other working hypotheses have also been proposed $[9,10,11]$. In the stochastic transport theory, RMP fields can drive the formation of magnetic island chains at rational surfaces in the plasma, where the safety factor $q$ is a rational number $m / n$. If these magnetic islands are wide enough and close enough to other island chains, then a stochastic magnetic field is created in the overlapping regions. The stochastic field enhances transport in these regions, lowering the pressure gradient and reducing the drive for ELMs. The extent of stochasticity is characterised by the Chirikov parameter, which is dependent on the local geometry, and on the pitch aligned components of the perturbation, as described in the cylindrical approximation for a given rational surface by the formula following [12].

$$
\sigma_{C h i r}=4 \sqrt{\left|\frac{h q^{2} R}{r_{s}} \frac{b_{m=n q}}{B}\right|}
$$

In the above, $q$ is the safety factor, $R$ the major radius, $B$ the equilibrium magnetic field, $h=$ $d(\log q) / d(\log r)$ is the normalised shear, $r_{s}$ is the minor radius and $b_{m=n q}$ the pitch aligned component of the magnetic perturbation. All quantities evaluated at the given rational surface.

Since the Chirikov parameter is proportional to the square root of the pitch aligned components, it is thought that maximising the pitch aligned components may lead to the largest stochastic region, and maximise the effect of the RMPs on the plasma. RMP experiments are therefore motivated to chose parameters which maximise the pitch aligned components of the perturbation. The total amplitude of the perturbation can be controlled by varying the coil currents, and the perturbation spectrum can also be modified; the dominant toroidal mode number $n$ can be chosen (from a small range) and the poloidal spectrum can be modified by changing the toroidal phase offset between the upper and lower coil sets, $\Delta \phi_{u l}$.

Numerical models are used to predict the pitch aligned RMP components for a given plasma equilibrium and applied perturbation. In many studies [3, 13, 14], experimental results of RMP experiments are interpreted assuming the 'vacuum approximation', that is, that the applied field perturbation is as it would be in the absence of a plasma. However, it has been shown that the 
plasma response to the applied perturbation can constitute a significant correction to the vacuum field $[15,16,17,18]$. Applied perturbations drive currents at rational surfaces in the plasma, which strongly suppress the pitch aligned components, and in the limit of zero resistivity, screen them completely. Furthermore, the applied perturbation can couple to and drive marginally stable modes in the plasma, a phenomenon known as resonant field amplification (RFA) [16]. In order to accurately calculate the stochasticity induced by an applied perturbation, the plasma response to the perturbation should be accounted for. A review of approaches to modelling the plasma response to applied perturbations is available in [19].

Previous investigations of the ideal single fluid plasma response to applied $n=2$ RMPs on the DIII-D tokamak [15], show a strong amplification of marginally stable kink modes, and suggest the possibility that this amplification may indirectly drive the pitch aligned components by 'spectral proximity' [20], ie, by having similar radial location and poloidal harmonic number, $m$. The specific mechanism could be poloidal harmonic coupling between the pitch aligned components and the amplified peeling response. It may be helpful to explicitly distinguish at this point between 'harmonic', which refers to a single poloidal fourier harmonic $m$ having poloidal dependence $e^{(i m \chi)}$ (where $\chi$ is the poloidal angle coordinate in the straight field line coordinate system), and 'mode', which refers to a particular type of plasma instability identified by its radial displacement profile, and is generally comprised of many poloidal harmonics. The 'kink response' refers to amplification of harmonics just above pitch resonance (ie, $m>$ $q n$ ). We also further divide the kink response into 'core kink' and 'low $n$ peeling' response, which refer to the kink response in the plasma core and

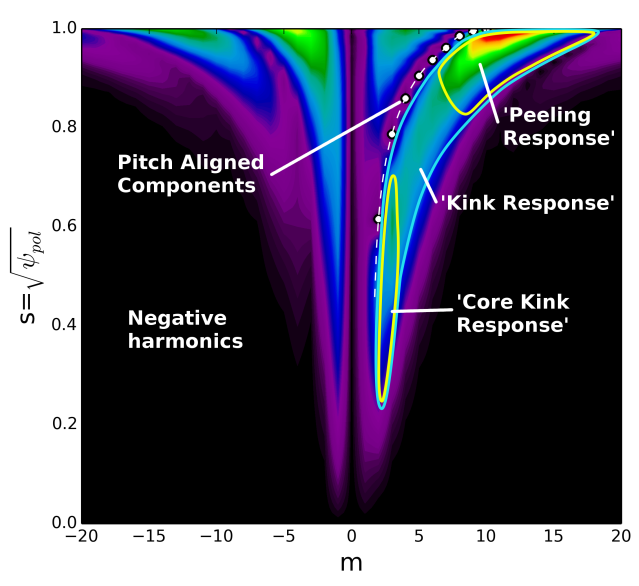

Figure 1. Spectrogram of the total field in response to an even coil configuration $\left(\Delta \phi_{u l}=0^{\circ}\right)$. The spectral regions (ie, regions in $m, s$ space) referred to as 'Kink', 'Core Kink' and 'Peeling' are labelled. More rigorously, the designations 'kink' and 'peeling' refer to specific radial profiles of the plasma displacement. The negative harmonics (ie, $m<0$ ) are of no interest to this study, since it is unlikely that these can influence the pitch aligned components. Note that $m$ is a discrete quantity, the $m$ axis is smoothed to make structures more easily discernible.

near the edge respectively. This nomenclature is illustrated in figure 1.

In this work, the plasma response to an $n=2$ RMP field applied to an ASDEXUpgrade plasma equilibrium is computed. The code MARS-F [21], which is well benchmarked against other codes [22] and validated against experiments [18, 23, 24], is used for the computation of the plasma response. MARS$F$ models the plasma response as a linear static 3D perturbation superimposed on a $2 \mathrm{D}$ equilibrium, and computes it by numerically solving the linearised perturbed equations of single fluid resistive MHD. The linear theory on which the MARS-F model built, is valid so long as the plasma displacement is sufficiently small compared with the equilibrium scale length. The limits of the validity are defined rigorously in [22]. The sensitivity of the plasma response to the geometry near the $\mathrm{X}$ point, is also investigated 
by varying plasma boundary shape. In order to investigate the general behaviour of poloidal harmonic coupling, a magnetic perturbation of a single poloidal harmonic is applied as a boundary condition at the plasma edge. In order to investigate specifically the effect of the peeling response on the pitch resonant components, a filtered boundary condition was designed, which contained only harmonics constituting the amplified peeling response. The pitch aligned components resulting from this applied boundary condition were then computed.

This paper is organised as follows. Section 2 describes the experimental equilibrium used for this study, and also how the applied perturbation is modelled and benchmarked. Section 3 introduces the nomenclature and components of the plasma response, and describes the computed plasma response to this applied perturbation, as well as its dependence on $\mathrm{X}$ point geometry. Section 4 describes the plasma response to customised boundary conditions, designed to investigate poloidal harmonic coupling. In section 5 some possible implications of these results are discussed.

\section{Equilibrium and Applied Perturbation}

\subsection{Equilibrium}

The 2D plasma equilibrium used in this study was reconstructed from an ASDEX-Upgrade experiment, shot number 30835, which was designed to study ELM mitigation at low collisionality. Essential information on the plasma equilibrium is listed in table 1 . The initial equilibrium reconstruction was done using the free boundary equilibrium code CLISTE [25]. Magnetic measurements, the $q=1$ surface location from measurements of sawtooth instabilities, and the scrape- off layer current were used as constraints. The equilibrium was then refined and mapped to the MARS-F straight field line coordinate system using the fixed boundary CHEASE [26] equilibrium solver. Figure 2 shows curves fitted to experimentally measured radial profiles of parameters which are also used as input for the MARS-F model: a) the electron temperature, b) ion temperature, c) plasma toroidal angular velocity, and d) the electron density.

\begin{tabular}{l|l|l|l} 
Shot num & Time & $q_{0}$ & $q_{95}$ \\
\hline 30835 & $3200 \mathrm{~ms}$ & 0.81 & 3.8 \\
& & & \\
$I_{p}(\mathrm{MA})$ & $B_{0}(\mathrm{~T})$ & $\beta$ & $T_{e, 0}, T_{i, 0}(\mathrm{keV})$ \\
\hline 0.77 & 1.7 & $1.9 \%$ & $6.96,5.05$
\end{tabular}

Table 1. Parameters of the plasma equilibrium of shot 30835 at $3200 \mathrm{~ms}$; the equilibrium used for this numerical study. $q_{0}$ and $q_{95}$ are the safety factor at the core and the $\Psi_{N}=0.95$ flux surface respectively. $I_{p}$ is the total plasma current, $B_{0}$ is the vacuum equilibrium field strength at the magnetic axis, $\beta=\left(2 \mu_{0}<P>\right) /\left(B_{0}^{2}\right)$ is the ratio of volume averaged plasma pressure to magnetic pressure on axis, $T_{e, 0}$ and $T_{i, 0}$ are the electron and ion temperatures at the magnetic axis.
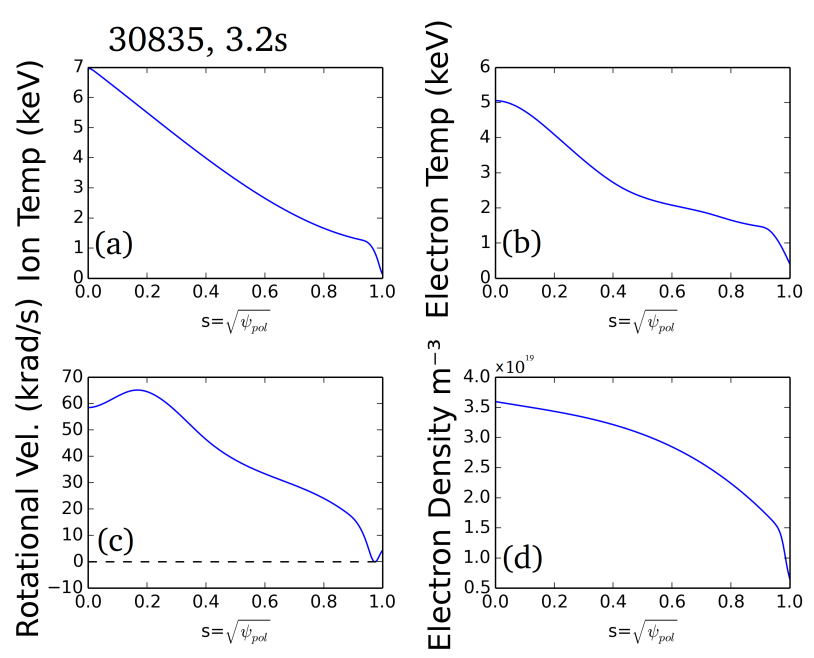

Figure 2. Ion and electron temperature, electron density and rotational velocity, from ASDEX-Upgrade shot 30835, taken at $3200 \mathrm{~ms}$ into the plasma shot. 


\subsection{Perturbation}

In the experiment, two coil sets, the upper and lower set each consisting of 8 coils, were used to apply a static magnetic perturbation to this equilbrium, with dominant toroidal mode number $n=2$. Figure 3 is a sketch of the AUG coil set and plasma boundary. The RMP coils are modelled as current perturbations, with deltalike functions of finite width at the poloidal locations of the coils, with a prescribed $e^{(i n \phi)}$ toroidal dependence. In the toroidal direction, a discrete set of 8 coils is modelled as a continuous sine wave with a single toroidal harmonic, $n$. The poloidal spectrum in contrast, contains many poloidal harmonics, $m$. With this given equilibrium and static perturbation, MARS-F can be used to solve the single fluid resistive MHD equations for the plasma response, that is, the state vector of perturbation quantities caused by the applied magnetic perturbation.
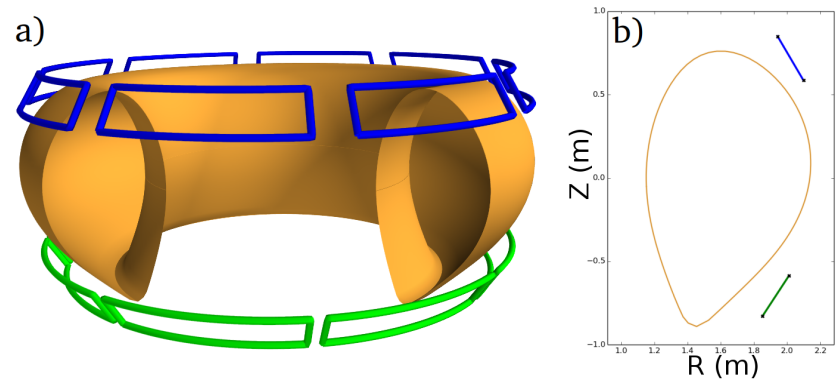

Figure 3. a) Sketch of the upper (blue) and lower (green) AUG RMP coil sets, in relation to the plasma boundary for shot 30835 (orange). b) A cross section showing the plasma boundary (orange), and the locations of the upper and lower coil sets.

\subsection{Benchmark}

In order to perform a benchmark of the vacuum field, the vacuum perturbation was first computed using MARS-F, and also separately using the Biot-Savart based ERGOS code [?]. The vacuum pitch aligned field components computed with MARS-F and ERGOS differ by less than $2 \%$. This vacuum benchmark gives confidence about the representation of the applied perturbation in MARS-F. A more detailed explanation and validation of the coil representation used in MARS-F can be found in [15].

\section{Plasma Response to Applied Perturbation}

The single fluid MHD plasma response was computed including plasma toroidal rotation, realistic geometry (excluding $X$ point), and resistivity, which was calculated with the Spitzer model (ie, $\eta \propto T_{e}^{-3 / 2}$ ). Since MARS-F is a linear model, the principle of superposition can be applied, and the response due to the upper and lower coil sets were computed separately. The total plasma response to both coils can then be computed in post-process for any $\Delta \phi_{u l}$ using the relation $b_{\Delta \phi_{u l}}=b_{\text {upper }}+b_{\text {lower }} \times e^{\left(i \Delta \phi_{u l}\right)}$, as is done in previous studies of the linear plasma response [15]. Figure 4 shows the vacuum perturbation, and the total magnetic perturbation including plasma response, ie, $b_{\text {total }}=b_{v a c}+$ $b_{\text {response. }}$ In this work, 'total' is taken to mean 'including the plasma response'. The quantity plotted is the absolute value of the normal component of the perturbed magnetic field, $\left|b^{1}\right|=$ $\left|\frac{\mathbf{b} \cdot \nabla \psi}{\mathbf{B}_{\text {eq }} \cdot \nabla \phi} \frac{q}{R_{0}^{2} B_{0}}\right|$. Several features are of interest. Firstly, at the inner rational surfaces the total pitch aligned components $\left(b_{m=q n}^{1}\right)$ are almost zero. This is because in the bulk plasma the plasma response is close to ideal, and so the pitch aligned components are almost perfectly screened by internal currents. However, closer to the plasma edge the temperature is lower, and so resistivity is higher, allowing the pitch resonant components near the plasma edge to be finite. Secondly, there are some areas of the spectrogram 

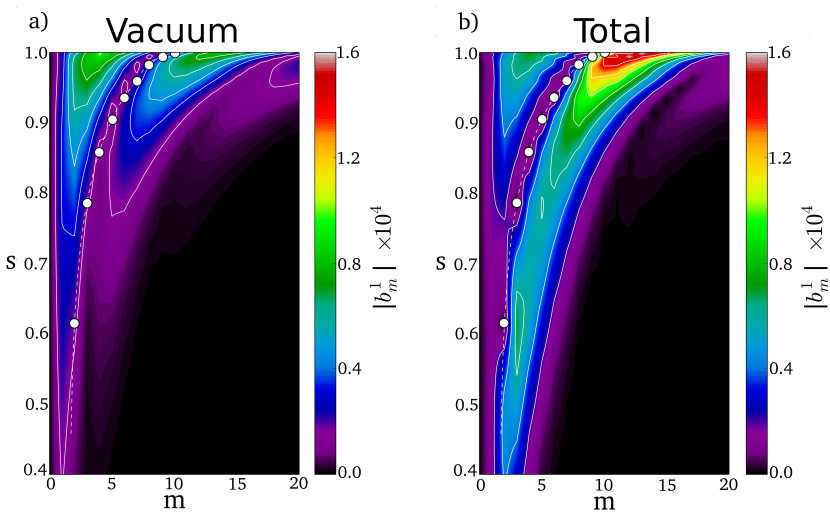

Figure 4. a) The poloidal spectrum of the applied field in the vacuum approximation, with $\Delta \phi_{u l}=180^{\circ}$. The pitch aligned components are highlighted with white circles, and the white dashed line follows the $m=n q(s)$ contour. b) The poloidal spectrum of the applied field including the plasma response (ie, the total field). There is some amplification of the core kink, but the edge localised peeling response is far more prominent. The pitch aligned components in the plasma bulk are completely screened by the plasma response, but the components close to the edge can remain finite.

which are amplified above their vacuum values by resonant field amplification. As figure 4 shows, the plasma response causes some amplification of the core kink mode, but the edge localised low $n$ peeling mode is the dominant response.

To measure the dependence of the peeling response on $\Delta \phi_{u l}$, the field amplitude at a representative spectral point (ie, a point in $m, s$ space) is computed for a scan of $\Delta \phi_{u l}$. Figure 5 shows the magnetic field perturbation $\left|b^{1}\right|$ at spectral location $(m, s)=(11,0.99) . s$ is the radial coordinate, defined as $s=\sqrt{\psi_{N}}$ where $\psi_{N}$ is the poloidal magnetic flux normalised such that $\psi_{N}=1.0$ at the plasma edge. The plot also shows the vacuum field amplitude at this spectral location. The figure shows that the plasma response amplifies the field in this spectral region far above its vacuum value. It also demonstrates the strong dependence of the response on the coil phase shift $\Delta \phi_{u l}$. This finding is qualitatively consistent with a similar

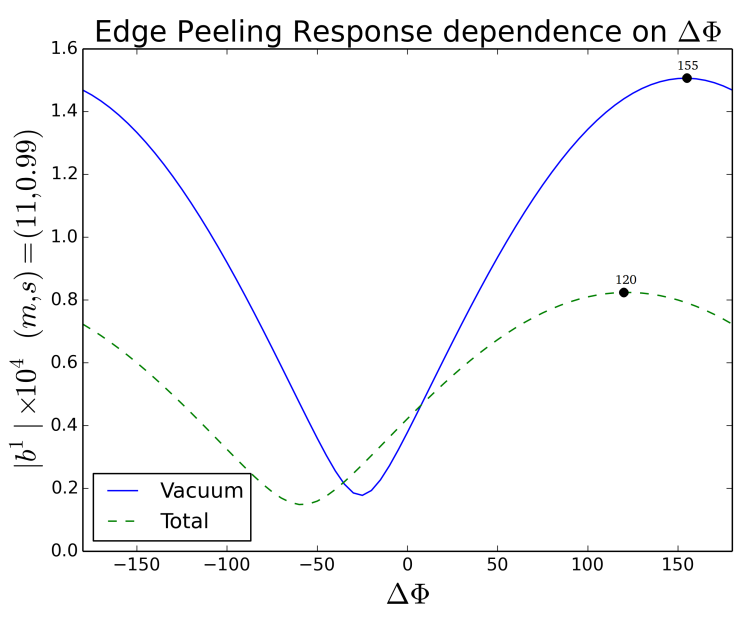

Figure 5. The magnitude of the perturbation at a specific spectral location $(m, s)=(11,0.99)$, which is representative of the amplified peeling response, with a scan of $\Delta \phi_{u l}$. The solid line shows the vacuum perturbation, and the dashed line is the total perturbation.

study of a DIII-D plasma, which also predicted amplification of the low $n$ peeling response with strong $\Delta \phi_{u l}$ dependence [15].

\subsection{Pitch Aligned Components}

The pitch aligned field components, marked as white circles in figure 1 , refer to spectral points (in $m, s$ space) where $m=n q(s)$. The pitch aligned field components are of particular interest to RMP studies, because they determine the width of any stochastic regions which may form in response to the RMPs. Figure 6 shows the magnitude of the vacuum (blue) and total (green) pitch aligned field components, computed with a coil phase difference of $\Delta \phi_{u l}=120^{\circ}$. In Ideal MHD, RMPs drive currents at rational surfaces which completely screen the pitch aligned components. This is why in the bulk plasma, where the resistivity is low, the plasma is approximately ideal and pitch aligned components tend to be very small. However, close to the plasma edge (roughly $s>0.95$ ) 


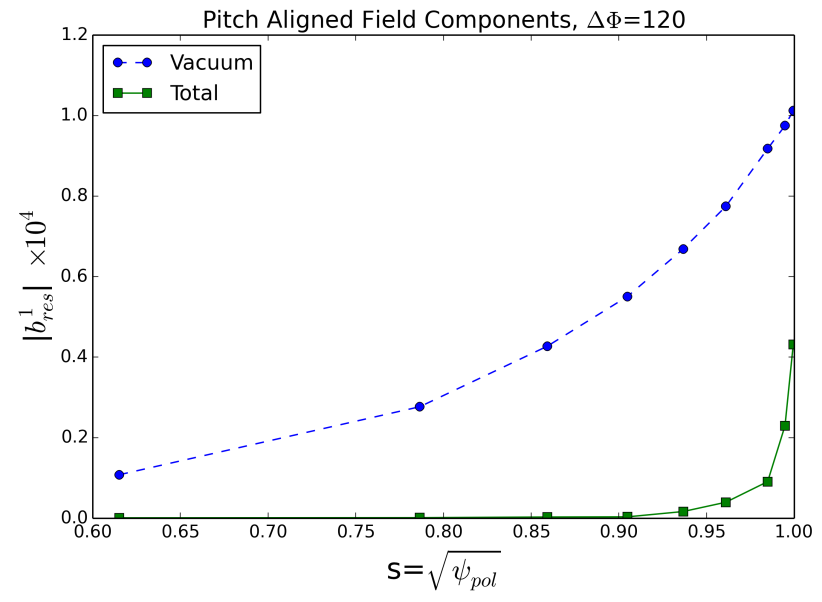

Figure 6. The pitch aligned components of the applied perturbation. In the plasma bulk, the pitch aligned components are well screened, but can be finite in the edge region where resistivity is higher.

the resistivity is much higher, so the pitch aligned components can be finite. In the plasma bulk and most of the edge region where the electron temperature is high, the Spitzer model for resistivity is quite acceptable. However, in the limit approaching the plasma edge where the electron temperature can tend to zero, the Spitzer resistivity would tend towards infinity. In the current scheme a numerical singularity is avoided by fixing a maximum value of the resistivity, chosen for numerical stability. A more refined model of resistivity at the plasma edge may include kinetic effects [27].

Figure 7 shows the amplitude of the outermost 3 pitch aligned components (at the $m=8$, 9 , and 10 surfaces) as a function of coil phase difference $\Delta \phi_{u l}$. The dashed lines show the pitch aligned components of the vacuum field, whereas the solid lines show the total field. The maximum value of the vacuum pitch aligned components (ie, vacuum alignment) occurs at around $\Delta \phi_{u l}=30^{\circ}$, whereas the maximum value of the total pitch aligned components occurs at around $\Delta \phi_{u l}=90^{\circ} ;$ the coil phase which maximises the

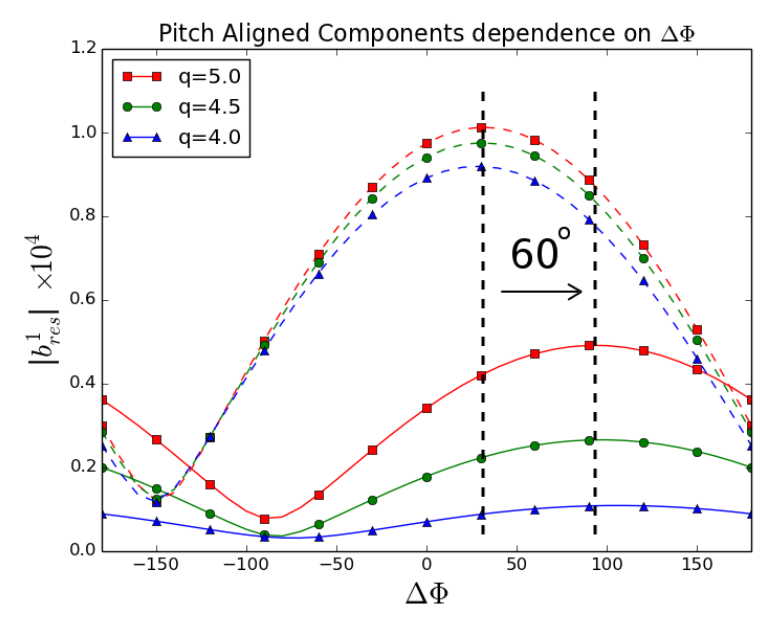

Figure 7. Magnitudes of the outermost 3 pitch aligned components, in the vacuum approximation (dashed lines) and the total field (solid lines). The maximum field including the plasma response is offset $60^{\circ}$ from its vacuum value. This tells us that vacuum modelling alone is insufficient to predict the coil phase for optimum pitch alignment.

total pitch aligned components, is offset from its vacuum value by $60^{\circ}$. This demonstrates that we may not expect a vacuum pitch aligned applied field to maximise the total pitch aligned components. Therefore in order to truly optimise the coil configuration to maximise total pitch aligned components, the plasma response must be accounted for. It should be noted that this phase shift is not specific to the resonant components. Non-resonant components also experience a shift in their dependence on $\Delta \phi_{u l}$ (see figure 5 ), but this effect will not be examined here. The $\Delta \phi_{u l}$ dependence of the total and vacuum fields is robust with respect to changes in $q_{a}$ caused by changes in plasma shape, and hence so is the $60^{\circ}$ offset of the total field from the vacuum. However the magnitudes of the outermost pitch aligned components were found to be slightly sensitive to $q_{a}$. The rotational and resistive radial profiles are fixed with respect to changes in $q_{a}$, but altering the edge $q$ profile changes the radial locations of the rational surfaces. Altering $q_{a}$ can therefore 
change the values of resistivity and plasma rotation at the pitch aligned components, which may explain this sensitivity.

Another interesting feature of figure 7 , is that for certain ranges of $\Delta \phi_{u l}$ the total pitch aligned components are of the same order or larger than their vacuum values, which is surprising considering the strong screening effects which act to reduce them. In the context of this linear single fluid MHD model, the mechanism by which parts of the perturbation spectrum could exceed their vacuum value, is resonant field amplification (RFA). It has previously been proposed [20], that the amplified edge localised peeling response may be indirectly driving the pitch aligned components by spectral proximity. That is, poloidal harmonics of the amplified peeling response may couple to and drive the pitch aligned components by poloidal harmonic coupling. This possibility motivated the investigation into poloidal harmonic coupling on ASDEX-Upgrade, described in section 4.

\subsection{Robustness of peeling response with respect to $X$ point truncation}

In divertor experiments, the $q$ profile is not defined at the $\mathrm{X}$ point where there is zero poloidal field. In the MARS-F flux-based coordinate system, this would introduce a numerical singularity, and so a certain truncation scheme must be used to exclude the $\mathrm{X}$ point. Truncation effectively approximates the divertor configuration as a limiter configuration with an otherwise similar shape, imposing a finite $q$ at the plasma edge, $q_{a}$. It has previously been suggested [15] that the predicted amplification of the low $n$ edge localised peeling response may be sensitive to the truncation around the $\mathrm{X}$ point, in particular to the value of the edge safety factor $q_{a}$.
In order to test the robustness of the peeling response with respect to changes in geometry around the $\mathrm{X}$ point, the plasma response was recomputed as the plasma boundary incrementally approached a separatrix. Figure 8 shows the plasma boundaries and edge $q$ profiles of 5 equilibria, identical except for differing levels of truncation around the $\mathrm{X}$ point, and as a result slightly different edge $q$ profiles. Figure 9 shows the edge spectrograms computed for different values of $q_{a}$. The spectrograms show a distortion of the spectrum radially, but not a change in amplitude. Changing the edge $q$ profile moves the $m=n q(s)$ curve, and the spectrogram is distorted radially following its movement. This suggests that while the response at a given radial position $s$ is sensitive to changes in geometry, the response at a given value of $q$ is not. Figure 10 shows the edge radial profile of the $m=12$ harmonic of the total magnetic perturbation, for 5 values of $q_{a}$. The plots show that changing the geometry has no effect on the amplified peeling response when $q(s)$ is used as the radial coordinate, suggesting that altering the $q$ profile only radially distorts the structure of the peeling response, and does not affect its amplitude. This suggests that in the limit of including the $X$ point, the amplified peeling response would still be present. We point emphasise that this is a study of the amplified plasma response of stable edge peeling modes, not the stability of these modes. Therefore this finding does not contradict previous studies [28] which find that the stability of edge peeling modes can be sensitive to the presence or absence of an $\mathrm{X}$ point.

The sensitivity of the plasma response to resistivity and plasma rotation, is as described in previous studies [16], and will not be discussed here. 

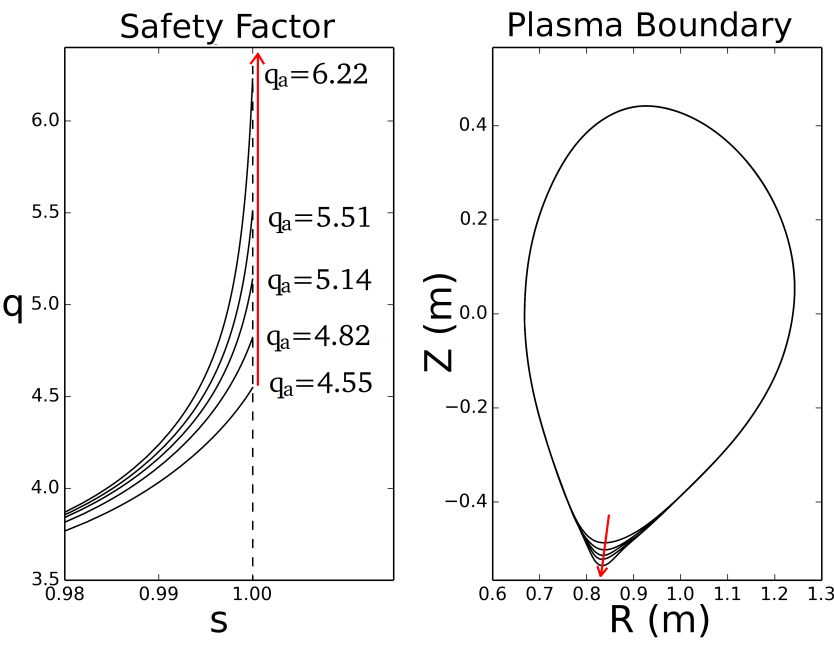

Figure 8. Plasma boundaries and $q$ profiles incrementally sharpening the $\mathrm{X}$ point, approaching a separatrix. A sharper (ie, less truncated) $\mathrm{X}$ point leads to higher values of $q_{a}$ in the equilibrium construction.

\section{Poloidal Harmonic Coupling on AUG}

\subsection{Generic poloidal coupling; single harmonic boundary condition}

Analytically, there are 3 primary sources of poloidal harmonic coupling: toroidicity couples harmonics with $\Delta m=\left|m-m^{\prime}\right|=1$, ellipticity couples harmonics with $\Delta m=2$, and triangularity couples harmonics with $\Delta m=$ 3. Since the MARS-F code operates in Straight Field Line (SFL) coordinates, geometrical and physical quantities are inseparable, so poloidal coupling can in principle occur between any pair of poloidal harmonics, and manifests both in the vacuum field, and the plasma response.

In order to investigate the generic behaviour of poloidal coupling in the MARS-F model, the code was used in an unconventional manner, in which the perturbation was not applied by RMP coils, but by a prescribed magnetic perturbation $b_{B C}^{1}$, applied at the plasma boundary. The perturbation at any closed surface (eg, the plasma boundary) completely determines the

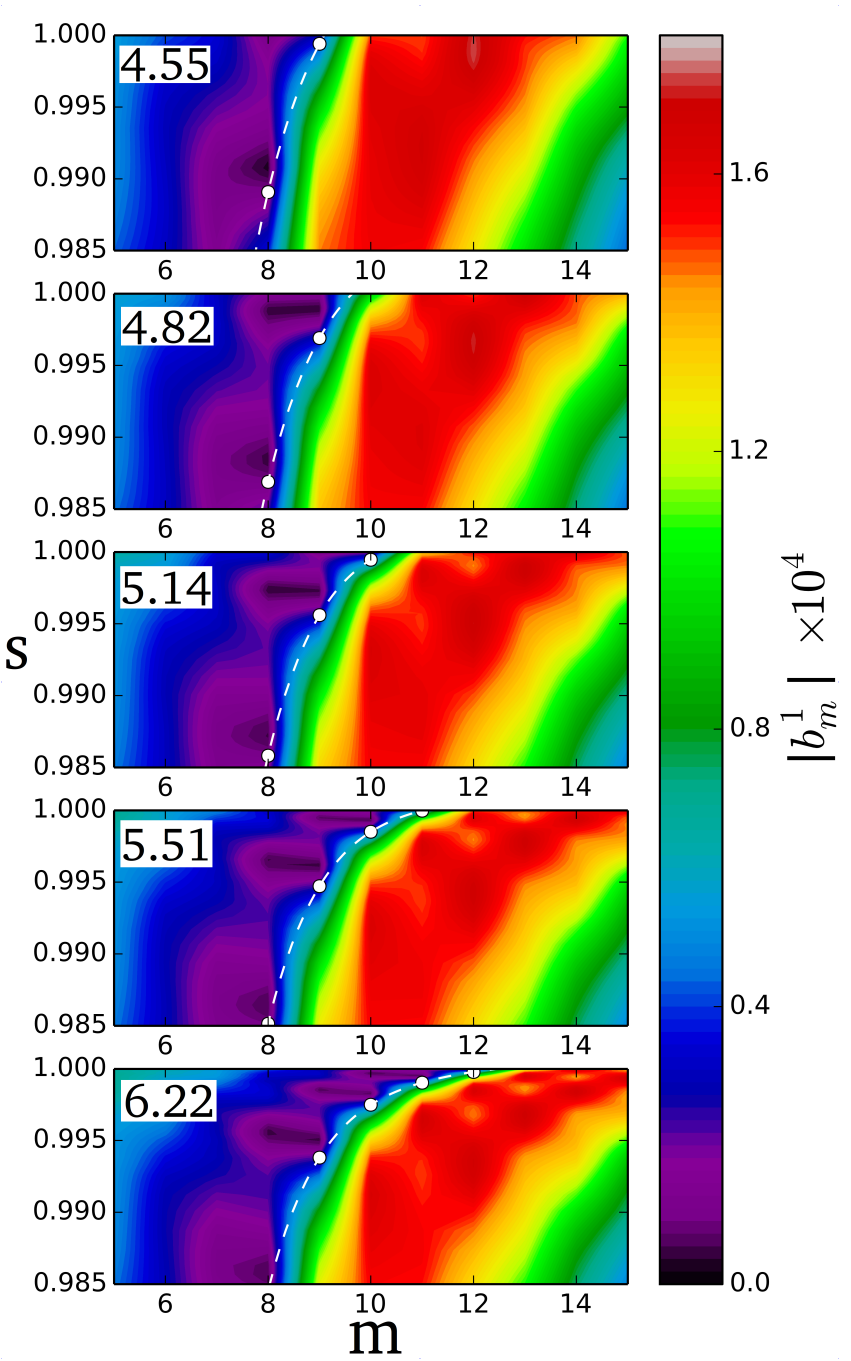

Figure 9. Edge regions of spectrograms from 5 different geometries, corresponding to 5 values of $q_{a}$. Increasing $q_{a}$ moves the $m=q n$ line, 'compressing' the peeling response structure radially, but not altering its global amplitude.

perturbation inside that surface, subject to the same physics as a normal RMP coil calculation. A magnetic perturbation with a single poloidal harmonic $m_{B C}$ and unit amplitude $\left(b_{B C}^{1}=1.0\right.$ for $m=m_{B C}$, and zero for $m \neq m_{B C}$ ) was applied at the plasma boundary, and the resulting magnetic field in the plasma bulk was computed. Figure 11 shows a spectrogram of a vacuum field and total field, resulting from a unit amplitude $m_{B C}=7$ and $m_{B C}=13$ magnetic perturbation applied at the plasma edge. At the plasma edge, the perturbation is purely single 

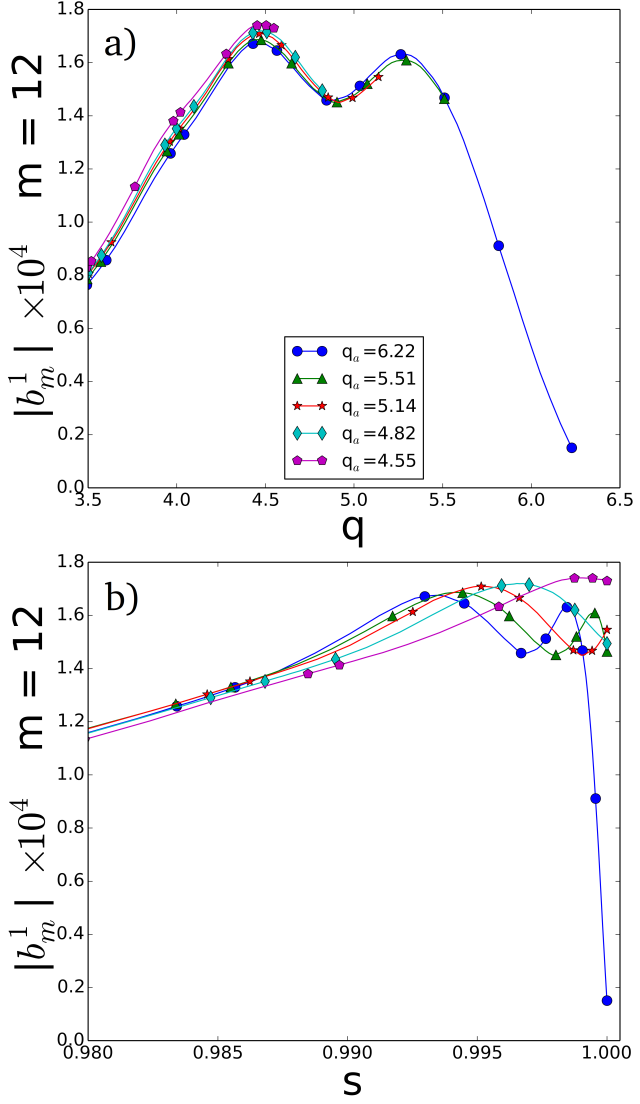

Figure 10. The edge radial profile of the $m=12$ harmonic of the total magnetic perturbation, using a) $q$ as radial coordinate, b) $s$ as radial coordinate. The plot shows that when $s$ is used as a radial coordinate, the peeling response appears to be sensitive to the $\mathrm{X}$ point truncation. However when $q$ is used as the radial coordinate, the edge profiles coincide up to each value of $q_{a}$.

$m$, but moving radially inwards, the spectrum broadens by poloidal harmonic coupling, and also moves to lower $|m|$. This behaviour appears to be general for any $m$. Figure 12 shows the total pitch aligned components, resulting from boundary perturbations with $m_{B C}=11,12$ and 13. For these computations, $m_{B C}$ were chosen such that they had no rational surface in the plasma. This means that the pitch aligned components can be non-zero only by poloidal coupling. The results show that a unit amplitude single $m$ perturbation applied at the plasma boundary, can drive the pitch aligned components

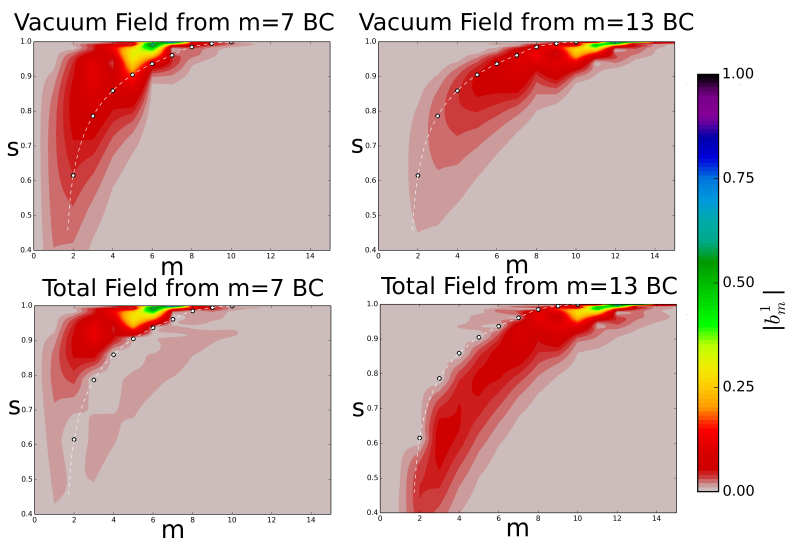

Figure 11. Spectrograms showing the perturbation resulting from applying a unit amplitude single $m_{B C}=7$ and $m_{B C}=13$ perturbation as a boundary condition at the plasma edge. Vacuum field and total field are shown. The spectrum broadens and shifts towards lower $|m|$ as it penetrates into the plasma bulk. Note that for clarity the colormap in these plots is reversed with respect to previous spectrograms.

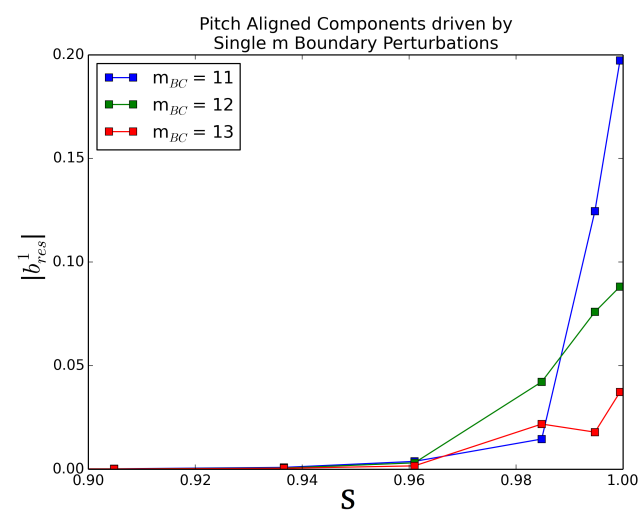

Figure 12. The total pitch aligned components of fields resulting from $m_{B C}=11,12$, and 13 unit amplitude boundary perturbations. The magnitude of the pitch aligned components is up to $20 \%$ of the applied unit field. Since there are no $m=11,12$ or 13 surfaces in the plasma, these pitch aligned components are non-zero only by poloidal harmonic coupling.

to be quite large relative to the size of the applied perturbation (in this work, up to $\left|b_{m=n q}^{1}\right| /\left|b_{B C}^{1}\right|=$ 0.2 ). This result suggests that it is quite possible for the pitch aligned components to be driven by poloidal harmonic coupling. 


\subsection{Amplified Peeling Response Boundary Condition}

By careful choice of boundary condition applied at the plasma edge, the question of whether the amplified peeling response could be driving the pitch aligned components on ASDEX-Upgrade 30835 , can be directly studied. The value of the magnetic perturbation due to the RMP coils and plasma response was computed in section 2 (figure 4b). This total perturbation was then filtered such that only harmonics which constitute the peeling response remain (ie, $m>11$ in this case), and then this filtered perturbation was used as the boundary condition applied at the plasma edge. Applying this boundary condition $b_{B C}^{1}=$ $b_{\text {peeling }}^{1}$ isolates the peeling response, allowing us to investigate its effects.

A simple check of the validity of this technique is to remove the filtering step before applying the boundary condition, which should recover the amplified peeling response predicted by the RMP computation. It was found that the unfiltered boundary condition exactly recovered the result from the full spectrum RMP computation, as expected.

Figure 13 shows the spectrograms of the vacuum and total fields, resulting from applying the peeling boundary condition, computed for $\Delta \phi_{u l}=0^{\circ}$ and $\Delta \phi_{u l}=180^{\circ}$. All spectrograms demonstrate the same shifts and broadening in the poloidal spectrum as seen in the single $m$ boundary condition computations (figure 11). Figure 14 shows the vacuum and total pitch aligned components resulting from the applied peeling response boundary condition, and compares them to the full spectrum computation. The figure shows that the pitch aligned components due to poloidal coupling with the amplified peeling response, can be

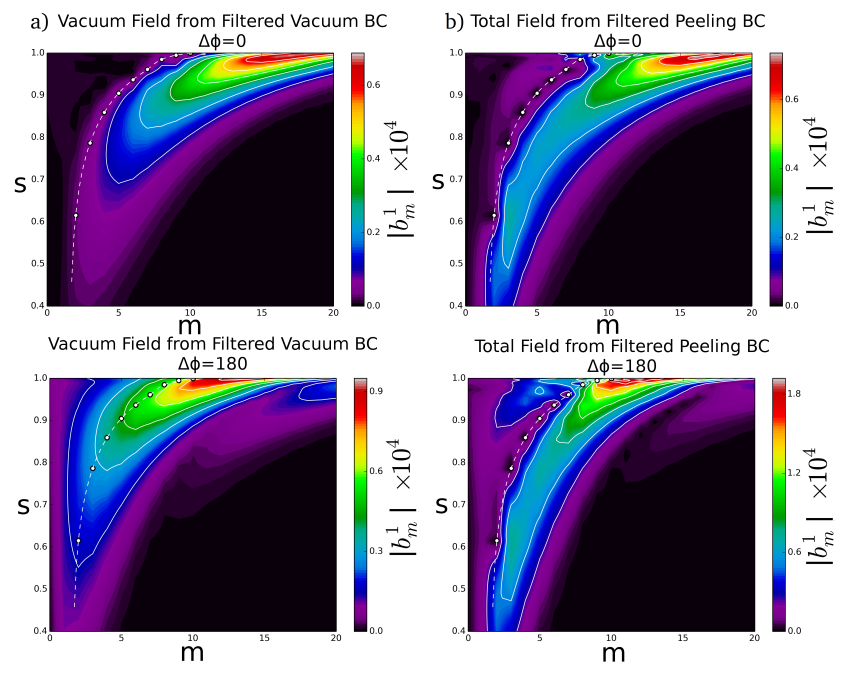

Figure 13. Spectrograms showing the perturbation in the plasma bulk, resulting from applying a boundary condition consisting of the amplified peeling response at $\Delta \phi_{u l}=0^{\circ}$ and $\Delta \phi_{u l}=180^{\circ}$. a) vacuum field, b) total field.

comparable to or larger than the pitch aligned components from the full spectrum. This result further suggests that the amplified peeling response can strongly affect the pitch aligned components, by poloidal harmonic coupling.

\section{Summary and Discussion}

Using the MARS-F code, the resistive single fluid plasma response to applied static RMP fields on AUG was investigated. Numerical computations predict a strongly amplified low $n$ peeling response, localised near the edge in the $s>0.7, m>q n$ region of the spectrum. The amplified peeling response showed no tendency to be reduced as the plasma boundary approached $\mathrm{X}$ point geometry (ie, for increasing $q_{a}$ ), rather the peeling response was distorted in the radial direction in response to the changing edge $q$ profile. That is, the peeling response is not sensitive to the numerical truncation near the $\mathrm{X}$ point when plotted as a function of $q$. It is also predicted that with finite resistivity, the outermost 

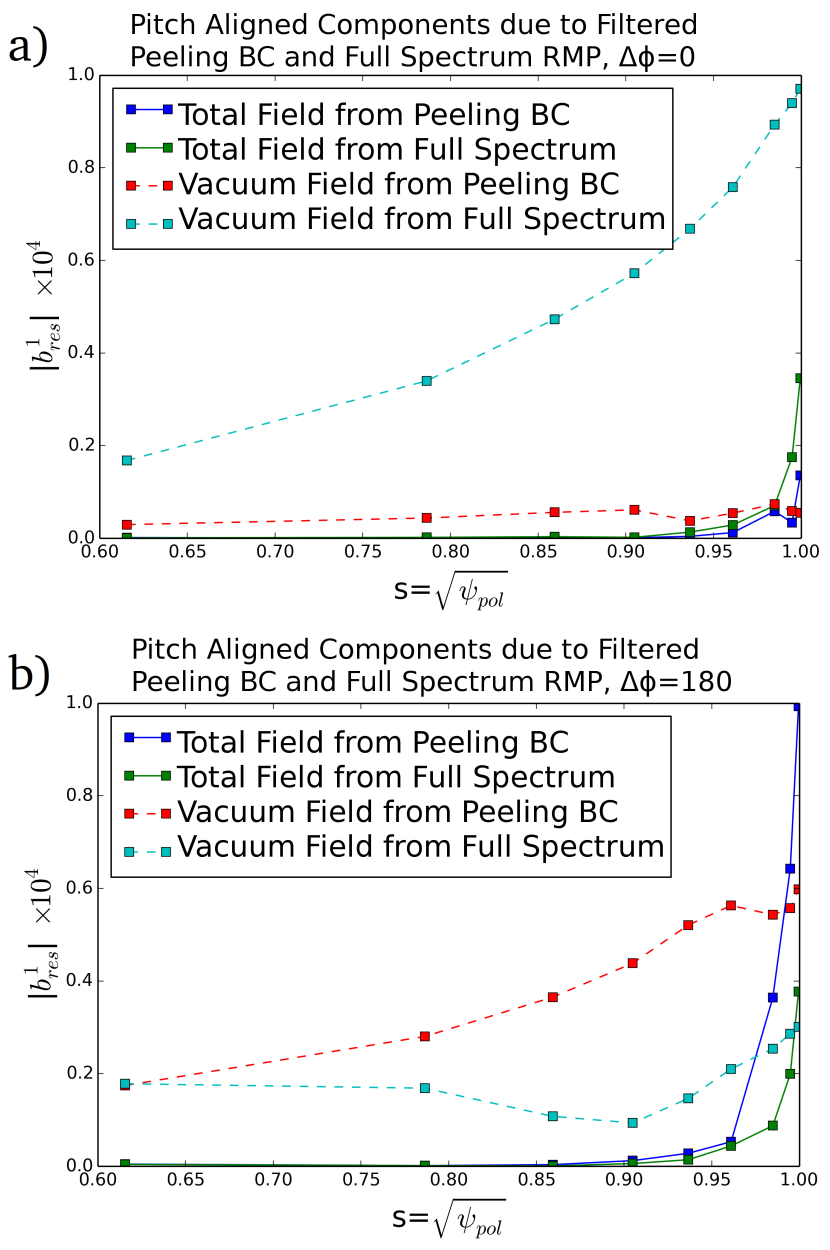

Figure 14. The pitch aligned field components resulting from applying the filtered peeling boundary condition, and also from the unfiltered RMP coil computation, for a) $\Delta \phi_{u l}=0^{\circ}$ and b) $\Delta \phi_{u l}=180^{\circ}$. The dashed lines denote the vacuum field, whereas the solid lines include the total field. The plots, in particular plot b), show that the applying the filtered peeling boundary condition drove the pitch aligned components to be large compared to their values in a full spectrum RMP coil computation.

pitch aligned components can be finite and comparable to the vacuum values. Both the pitch aligned components and the peeling response have a strong dependence on upper/lower coil phase difference $\Delta \phi_{u l}$. Also, the value of $\Delta \phi_{u l}$ at which the total pitch aligned components are maximised, is offset from optimum vacuum pitch alignment by $60^{\circ}$. This result highlights the importance of including the plasma response when calculating optimum coil phase for future RMP experiments. A vacuum calculation alone would not predict the correct coil configuration for maximum edge stochasticity. A comparison between the MARS-F modelling results and the experimental scans of $\Delta \phi_{u l}$ in ASDEX-Upgrade has been partially performed in [29], and a systematic comparison will be carried out in the future.

To investigate the proposal that the amplified peeling response could drive the pitch aligned components by poloidal harmonic coupling, a study of poloidal coupling on AUG was undertaken. To demonstrate general poloidal harmonic coupling in realistic tokamak geometry, single $m$ perturbations were applied as boundary conditions at the plasma edge, and the resulting bulk perturbation computed. The results showed that even when perturbations have only a single poloidal mode number at the plasma surface, in the plasma bulk the spectrum broadens by poloidal harmonic coupling, and also shifts towards lower $|m|$. Computations of the plasma response also showed that poloidal harmonic coupling could drive the total pitch aligned components. To isolate the effect of the amplified peeling response on the pitch aligned components, the amplified peeling response was prescribed as a boundary condition with other harmonics filtered out, and the resulting pitch aligned components computed. The computations predict that the amplified peeling response can drive the pitch aligned components by poloidal harmonic coupling in some circumstances.

In experiments it is possible to measure the peeling response using external magnetic pickup coils [30], and it is often possible to measure magnetic island widths using Thomson Scattering [31] or Electron Cyclotron Emission [32], from which the pitch aligned field components 
can be calculated. If the pitch aligned components are being driven primarily by the amplified peeling response, then we may expect to find a correlation between the measured peeling response and the measured island widths. However, if the peeling response were varied via an experimental scan of $\Delta \phi_{u l}$, then it is possible that a correlation may not be expected. This is because the total field is the sum of the plasma response and vacuum field, which have dependencies on $\Delta \phi_{u l}$ offset from each other. However, a scan of $q_{95}$ for fixed vacuum field may change the amplitude of the peeling response independently of the vacuum field.

If a large stochastic edge region is an important component of RMP effects on tokamak plasmas, then it follows that the RMP configuration which should have the largest effect on the plasma is the one which maximises the pitch aligned components, and hence the stochastic region. These results imply that the optimal coil configuration for stochasticity, may depend closely on the amplified peeling response, rather than solely the vacuum field. This may be a useful consideration when designing future RMP experiments, and further motivates the creation of plasma response 'parameter maps', such as was calculated for DIII-D [15].

\section{Acknowledgements}

This work is part-funded by the EPSRC through the Fusion Doctoral Training Network (grant number EP/K504178/1), and part-funded by the RCUK Energy Programme (under grant EP/I501045) and the European Communities. To obtain further information on the data and models underlying this paper please contact PublicationsManager@ccfe.ac.uk. The views and opinions expressed herein do not necessarily reflect those of the European Commission.

\section{References}

[1] A. W. Leonard, 2014, Physics of Plasmas, 21, 90501.

[2] T. E. Evans et al, 2004, Physical Review Letters, 92, 235003.

[3] Y. M. Jeon et al, 2012, Physical Review Letters, 109, 035004.

[4] Y. Liang et al, 2007, Physical Review Letters, 98, 265004.

[5] A. Kirk et al, 2013, Nuclear Fusion, 53, 043007.

[6] W. Suttrop et al, 2011, Physical Review Letters, 106, 225004.

[7] P.T. Lang et al, 2013, Nuclear Fusion, 53, 043004.

[8] T. E. Evans et al, 2006, Nature Physics, 2, 419-423.

[9] Y. Liang et al, 2010, Physical Review Letters, 105, 065001.

[10] I.T. Chapman et al, 2012, Nuclear Fusion, 52, 123006.

[11] M. Bécoulet et al, 2014, Physical Review Letters, 113, 115001

[12] I. Joseph, 2012, Contributions to Plasma Physics, 52, 326-347.

[13] T. E. Evans et al, 2006, Physics of Plasmas, 13, 056121.

[14] T.E. E. Evans et al, 2008, Nuclear Fusion, 48, 024002.

[15] S. R. Haskey et al, 2014, Plasma Physics and Controlled Fusion, 56, 035005.

[16] Y. Q. Liu et al, 2010, Physics of Plasmas, 17, 122502.

[17] Y. Q. Liu et al, 2011, Nuclear Fusion, 51, 083002.

[18] M. J. Lanctot et al, 2010, Physics of Plasmas, 17, 030701.

[19] a.D. D. Turnbull, 2012, Nuclear Fusion, 52, 054016.

[20] M. J. Lanctot et al, 2013, Nuclear Fusion, 53, 083019.

[21] Y. Q. Liu et al, 2000, Physics of Plasmas, 7, 3681.

[22] A. D. Turnbull et al, 2013, Physics of Plasmas, 20, 056114.

[23] M. J. Lanctot et al, 2011, Physics of Plasmas, 18, 056121.

[24] Y. Q. Liu et al, 2012, Plasma Physics and Controlled Fusion, 54, 124013.

[25] P J Mc Carthy, 2011, Plasma Physics and Controlled Fusion, 54, 015010.

[26] H. Lütjens et al, 1996, Computer Physics Communications, 97, 219-260.

[27] G. Park et al, 2010, Physics of Plasmas, 17, 102503.

[28] G. T. Huysmans, 2005, Plasma Physics and Controlled Fusion, 47, 2107-2121.

[29] A Kirk et al, 2014, pre-print arXiv ID 1412.7631, 131.

[30] Y. Q. Liu et al, 2010, Plasma Physics and Controlled Fusion, 52, 045011. 
[31] K. Narihara et al, 2001, Physical Review Letters, 87, 135002.

[32] A Isayama et al, 1999, Plasma Physics and Controlled Fusion, 41, 35-43. 